\title{
ENVIRONMENTAL INFLUENCE ON THE OBSERVED RELAXATION BEHAVIOUR OF TWO CHROMIUM SYSTEMS
}

\author{
G. J. GERRITSMA, J. FLOKSTRA, G. A. HARTEMINK and A. de BLOK \\ Twente University of Technology, P.O. Box. 217, 7500 AE Enschede, The Netherlands
}

\begin{abstract}
EPR recovery curves were measured for $\mathrm{KCr}$ alum and ruby with $2.7 \%$ chromium. A considerable influence of the heat transport properties of the bath is observed. Taking care of this influence a spin relaxation time $\tau=(0.7 \pm 0.2) \mathrm{ms}$ could be observed for ruby and a spin-lattice relaxation time $T_{1} \propto T^{-1}$. For $\mathrm{KCr}$ alum an anomalous behaviour of $T_{1}$ was observed which may be due to a phonon bottleneck.
\end{abstract}

\section{Introduction}

We investigated the relaxation behaviour of single crystals of $\mathrm{KCr}$ alum and ruby with $2.7 \% \mathrm{Cr}$ by weight at liquid-helium temperatures with a pulsed EPR spectrometer which operates at X-band frequencies. At these temperatures the direct spin-lattice relaxation process dominates but at higher $\mathrm{Cr}$ concentrations phonon bottleneck effects are likely to occur and general cross relaxation effects also appear. Chromium concentration dependent relaxation effects in ruby and $K$ alum were for example investigated by Nisida [1] and Standley and Tooke [2]. At the lowest $\mathrm{Cr}$ concentrations in the spin-lattice relaxation time $T_{1}$ was proportional to $T^{-1}$ and at the highest concentrations to $T^{-2}$. This indicates that a direct process is effective, which is bottlenecked at the higher $\mathrm{Cr}$ concentrations. Cross relaxation effects were also observed.

In a previous paper [3] we showed that heat conduction in the bath may have a considerable influence on the shape of a recovery curve and hence the $T_{1}$ obtained from the tail of a curve is erroneous. Taking $T_{1}$, the thermal conductivity and the specific heat of the bath $\lambda_{\mathrm{g}}$ and $C_{\mathrm{g}}$, the ratio of the spin and the lattice specific heat $C_{\mathrm{s}} / C_{\mathrm{L}}$ and the crystal radius $r_{0}$ into account we solved the corresponding heat transport equations numerically. In the case that $C_{\mathrm{g}} \rightarrow 0$ a recovery curve can be expressed as a sum of two exponentials. By comparing measured and calculated curves we obtain $T_{1}$ and $C_{\mathrm{s}} / C_{\mathrm{L}}$.

\section{Experiments}

Experiments were performed with crystals having an effective diameter of 2 to $3 \mathrm{~mm}$. They were mounted in a cavity which could be filled with helium gas and by changing the pressure, $C_{\mathrm{g}}$ could be varied. The cavity was immersed in liquid helium in order to obtain the appropriate bath temperature. Recovery curves were measured as a function of bath pressure, temperature and saturation pulse width and height.

With ruby we performed three distinct series of measurements. The 1-3 high field transition was saturated with a $3 \mathrm{~ms}$ pulse. The magnetic field $H=2.7 \mathrm{kOe}$ made an angle of $20^{\circ}$ with the crystal axis. The bath temperature varied from 4.2 to $1.8 \mathrm{~K}$.

In the first series the crystal was directly immersed in liquid helium. From the tail of the recovery curves we obtained $T_{1}=12 T^{-1.6} \mathrm{~ms}$. This is in accordance with the results of ref. [2].

In the second series the bath pressure $p$ was 0.25 or 0.15 Torr. The recovery curves were decomposed into a sum of two exponentials $\left(C_{\mathrm{g}} \rightarrow 0\right)$.

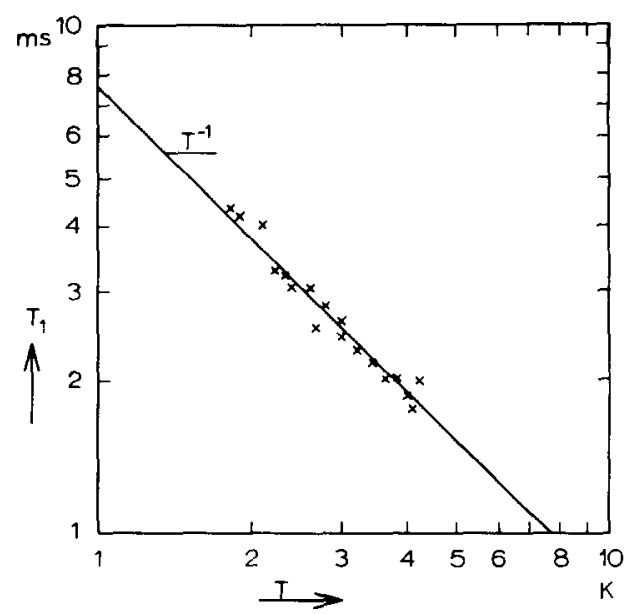

Fig. 1. Spin-lattice relaxation time $T_{1}$ of ruby showing a $T^{-1}$ behaviour. 
From the parameters thus obtained $T_{1}$ was calculated. The result is presented in fig. 1 and it can be seen that $T_{1} \propto T^{-1}$, which differs from the previous result. A temperature run was also made with $p=12$ Torr and using the numerical technique all curves could be fitted with $T_{1} \propto T^{-1}$ as above and $C_{\mathrm{s}} / C_{\mathrm{L}} \propto T^{-3}$.

In the third series $T=4.2 \mathrm{~K}$ and $p$ varied from 610 to 0.04 Torr. All curves could be fitted using values for $T_{1}$ and $C_{\mathrm{s}} / C_{\mathbf{L}}$ consistent with the results of the second series. Hence we conclude that $T_{1}$ is proportional to $T^{-1}$ and not to $T^{-1.6}$ even though the $\mathrm{Cr}$ concentration is rather high.

In all series mentioned above we ignored the first few milliseconds after the saturation pulse because our thermal conduction model failed to describe this part of the curves. To account for this, we assumed that the relaxation process can be divided into two distinct steps. First the spin system comes into internal equilibrium and then the relaxation towards the lattice and bath comes into effect. In all cases the initial stage of the relaxation process, corrected for heat loss to lattice and bath, is exponential with time constant $\tau=(0.7 \pm$ $0.2) \mathrm{ms}$. The amplitude of this part decreases with increasing saturation pulse width.

In fig. 2 relaxation times for $\mathrm{KCr}$ alum $(H=3.3$ kOe and $\nu=9.3 \mathrm{GHz}$ ) obtained from the initial slope of the recovery curves are given. Four different gas pressures were chosen. If $p=10$ or 30 Torr $T_{1}=28 T^{-1.5} \mathrm{~ms}$ whereas if $p=0.01$ or 1 Torr, $T_{1} \propto T^{-3}$. The latter result is in agreement with relaxation times obtained from low-frequency dynamic susceptibility measurements and with $\mathrm{cw}$ saturation measurements of Bölger et al. [4]. However, it is not clear why there is such a marked difference between the two groups of measurements, but in this case the phonon bottleneck could play an important role. We observed that the initial slope of a recovery curve increases with increasing

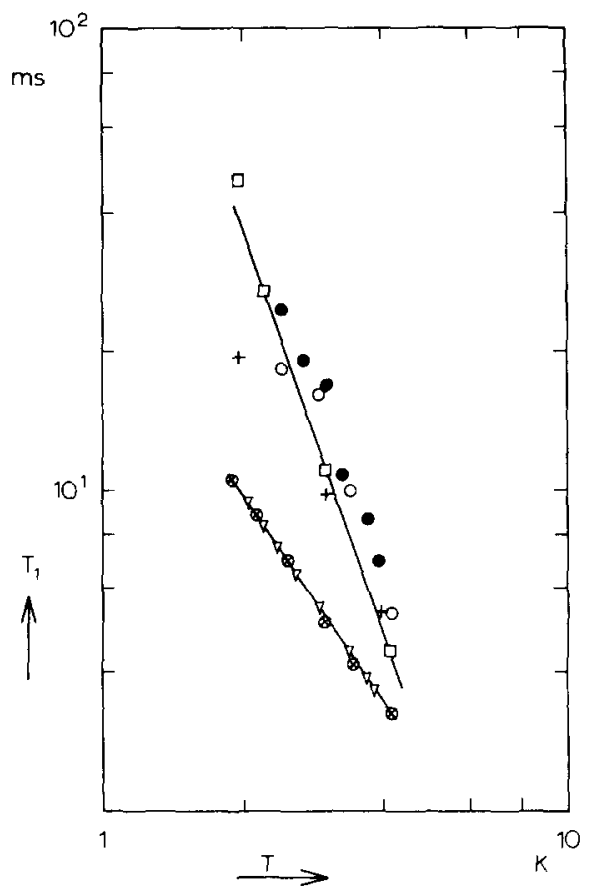

Fig. 2. Spin-lattice relaxation time $T_{1}$ of $\mathrm{KCr}$ alum showing an anomalous behaviour; $O, \bullet, \nabla$ and $\otimes$ : exchange gas pressure $p=0.01,1,10$ and 30 Torr, $\square:$ Lfr data, $+:$ cw data of Bölger et al.

saturation pulse width and height as long as the spin system was not fully saturated. A further increase does not affect the initial slope which is consistent with what is expected in the case of phonon bottleneck.

\section{References}

[1] Y. Nisida, J. Phys. Soc. Japan 19 (1964) 2273.

[2] K. J. Standley and A. O. Tooke, J. Phys. 1C (1968) 149.

[3] G. J. Gerritsma, J. Flokstra, G. A. Hartemink, J. J. M. Scholten, A. J. W. A. Vermeulen and L. C. van der Marel, Physica 95B (1978) 173.

[4] B. Bölger, J. M. Noothoven van Goor and K. J. van Damme, Physica 27 (1961) 18. 